\title{
New results on soft particle production in heavy-ion collisions with LHCb
}

\author{
Óscar Boente García*, on behalf of the LHCb Collaboration \\ Universidade de Santiago de Compostela, Spain \\ E-mail: oscar.boente@cern.ch
}

In this contribution, the latest measurements on soft particle production in ion and $p p$ collisions made by the $\mathrm{LHCb}$ experiment are reported. The proceeding addresses antiproton production in $p \mathrm{He}$ collisions, the measurement of Bose-Einstein correlations of same-charged pions in $p p$ collisions and the latest advances with prompt photon production in $p \mathrm{~Pb}$ at LHCb. All these measurements cover a wide range of physics cases, opening new horizons in heavy-ion physics at LHCb.

Sixth Annual Conference on Large Hadron Collider Physics (LHCP2018)

4-9 June 2018

Bologna, Italy

${ }^{*}$ Speaker. 


\section{Introduction}

The $\mathrm{LHCb}$ detector is a forward spectrometer located at the LHC covering the acceptance range in pseudo-rapidity of $2<\eta<5$. It is instrumented with a vertex locator (VELO), a tracking system with excellent $p_{\mathrm{T}}$ resolution, two ring-imaging Cherenkov detectors (RICH) that provide excellent charged hadron identification, calorimetry and a muon system [1]. The detector was designed for heavy-flavour measurements oriented towards searches of physics beyond the Standard Model as well as precision tests of the Standard Model. However, LHCb joined the LHC heavy-ion physics program, and since then its capabilities in this scenario have been explored. In particular, LHCb can operate in two different configurations: fixed-target mode and collider mode, covering a wide range of physics cases.

In its fixed-target configuration, $\mathrm{LHCb}$ becomes a fixed-target experiment by exploiting its forward geometry. The target is provided by injecting an inert gas in the LHC beam line at the $\mathrm{LHCb}$ interaction point [2]. Since 2015, data have been acquired in a variety of set-ups with energies in the nucleon-nucleon centre-of-mass system of $68.6 \mathrm{GeV}, 86.6 \mathrm{GeV}$ and $110.5 \mathrm{GeV}$ (see figure 1). With regard to the collider mode, LHCb completed several data-taking campaigns of proton-lead collisions (at $\sqrt{s_{\mathrm{NN}}}=5.02 \mathrm{TeV}$ in 2013 with $1.6 \mathrm{nb}^{-1}$ and $8.16 \mathrm{TeV}$ in 2016 with $34 \mathrm{nb}^{-1}$ ) and lead-lead collisions $\left(\sqrt{s_{\mathrm{NN}}}=5.02 \mathrm{TeV}\right.$ in 2015 with $\left.10 \mu \mathrm{b}^{-1}\right)$.

\section{Antiproton production in pHe collisions at $\sqrt{\mathbf{s}_{\mathrm{NN}}}=110.5 \mathrm{GeV}$}

PAMELA and AMS have determined the ratio antiproton/proton in cosmic rays with great precision, showing some hints on an excess with respect to the predictions at particle energies of the order of $100 \mathrm{GeV}[3,4]$. The measurement is known to be a sensitive indirect probe for exotic astrophysical sources of antimatter. In order to clarify the situation, the uncertainties of the theoretical prediction must be reduced, which can be accomplished by increasing the precision of the antiproton production cross-section in interactions of cosmic rays with the interstellar medium. In particular, an important contribution to the uncertainty comes from proton-helium scattering, for which there was no direct measurement at the moment.

Motivated by this experimental situation, LHCb performed a measurement of the antiproton cross-section in $p \mathrm{He}$ at $\sqrt{s_{\mathrm{NN}}}=110.5 \mathrm{GeV}$ using data acquired in 2016 in its fixed-target configuration [5]. ${ }^{1}$ In this analysis, the cross-section of antiprotons is determined in bins of $\left(p, p_{\mathrm{T}}\right)$.

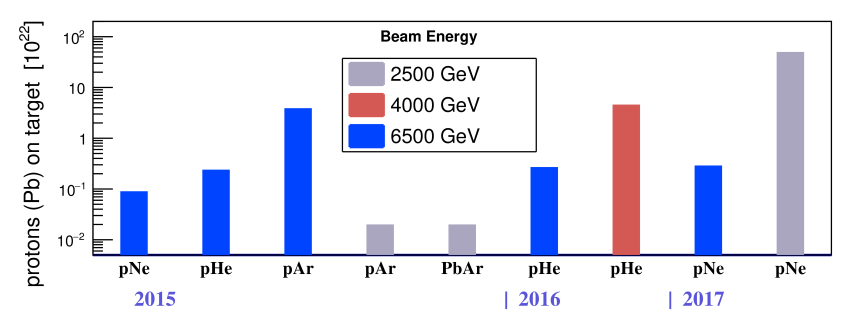

Figure 1: Diagram showing the different fixed-target configurations in which LHCb has acquired data up to date.

\footnotetext{
${ }^{1}$ The paper was not published at the time of the conference
} 

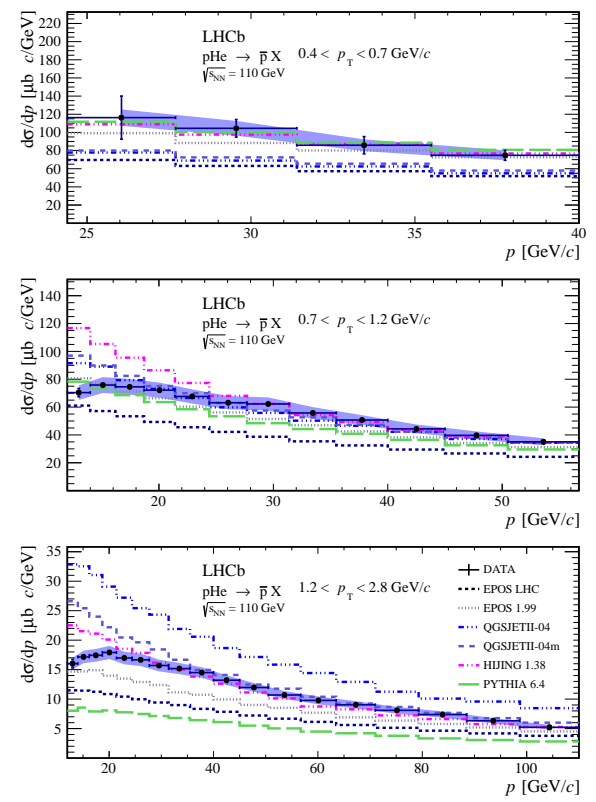

Figure 2: Antiproton production cross-section as a function of momentum, integrated over various $p_{\mathrm{T}}$ regions. The data points are compared with predictions from theoretical models. The uncertainties on the data points are uncorrelated only, while the shaded area indicates the correlated uncertainty [5].

Negatively charged particles were selected and antiprotons were discriminated from pions and kaons thanks to the excellent particle identification capabilities of the detector. Due to the lack of a precise measurement of the injected gas pressure, the luminosity was determined indirectly using the yield of electrons from elastic scattering of the proton beam. The key aspects of this procedure are the well known cross-section of the process and the possibility to isolate precisely this kind of events. Additionally, the background for non-prompt antiprotons and from interactions with the residual gas in the cavity was also considered and corrected.

Results for the double-differential antiproton cross-section are shown in figure 2, and are compared with the predictions from different simulation models: EPOS-LHC [6], EPOS 1.99 [7], HIJING 1.38 [8], PYTHIA 6.4 [9], QGSJETII-04 [10] and its low-energy extension QGSJETII-04m. The systematic uncertainties are smaller than $10 \%$ for most kinematic bins. The total yield of $p \mathrm{He}$ inelastic collisions which are visible in LHCb is determined from the yield of reconstructed primary vertices and is found to be compatible with EPOS-LHC: $\sigma_{\mathrm{LHCb}} / \sigma_{\mathrm{EPOS}-\mathrm{LHC}}=1.08 \pm 0.07 \pm 0.03$, where the first uncertainty is due to the luminosity and the second to the primary vertex (PV) reconstruction efficiency. This result indicates that the significant excess of antiproton production over the EPOS-LHC prediction seen in figure 2 is mostly due to the antiproton multiplicity.

\section{Bose-Einstein correlations of same-sign charged pions in pp at $\sqrt{\mathrm{s}}=7 \mathrm{TeV}$}

The LHCb experiment has measured Bose-Einstein correlations (BEC) in pairs of same-sign pions from $p p$ collisions at $\sqrt{s}=7 \mathrm{TeV}$ as a function of charged particle multiplicity [11]. This effect can be used to study multiparticle production within the process of hadronisation [12]. In 


\begin{tabular}{ccc}
\hline Activity class & $R[\mathrm{fm}] \pm$ stat \pm sys & $\lambda \pm$ stat \pm sys \\
\hline Low & $1.01 \pm 0.01 \pm 0.10$ & $0.72 \pm 0.01 \pm 0.05$ \\
Medium & $1.48 \pm 0.02 \pm 0.17$ & $0.63 \pm 0.01 \pm 0.05$ \\
High & $1.80 \pm 0.03 \pm 0.16$ & $0.57 \pm 0.01 \pm 0.03$ \\
\hline
\end{tabular}

Table 1: Results of fits to the double ratio $r_{d}(Q)$ for the three different activity classes, using the Levy parametrization. Statistical and systematic uncertainties are given separately.

particular, it allows to constrain the spatio-temporal characteristics of the particle emission patterns depending on the final-state particle multiplicity. The measurement constitutes the first of its kind at forward rapidities.

The observable is the correlation function: $C_{2}(Q)=\frac{N(Q)^{\mathrm{SAME}}}{N(Q)^{\mathrm{REF}}}$, where $Q=\sqrt{-\left(q_{1}-q_{2}\right)^{2}}$ is the absolute value of the four-momentum difference between pairs of same-charged pions and gives a measure of their phase-space separation. Here, $N(Q)^{\mathrm{SAME}}$ is the distribution extracted from pairs of signal particles, while $N(Q)^{\mathrm{REF}}$ is a reference data-driven sample. The signal pairs are defined as same-charged pions coming from the same PV. The reference sample is constructed with pairs of pions from different events, removing the BEC effect but maintaining other types of correlations. The study is performed using a double ratio $r_{d}(Q)$ of the correlation function in data and the same function in a simulated sample where BEC effect has been switched off to correct for imperfections in the reference sample. Coulomb interaction between final-state pions is not included in the simulation, so the Gamov penetration factor is introduced in data for correction [13].

Events are classified in multiplicity classes based on the number of tracks in the VELO, which is a good measure of charged-particle multiplicity $N_{c h}$ (see figure 3). The correlation function is parametrized following Levy [14] as $C_{2}(Q)=N\left(1 \pm \lambda e^{-R Q}\right) \cdot(1+\delta Q)$, where $\lambda$ is the chaoticity or partial incoherence of the source, $R$ is the correlation radius or size of the emission volume and $\delta$ parametrizes long range correlations. The double ratio for each multiplicity class is fitted to this parametrization.

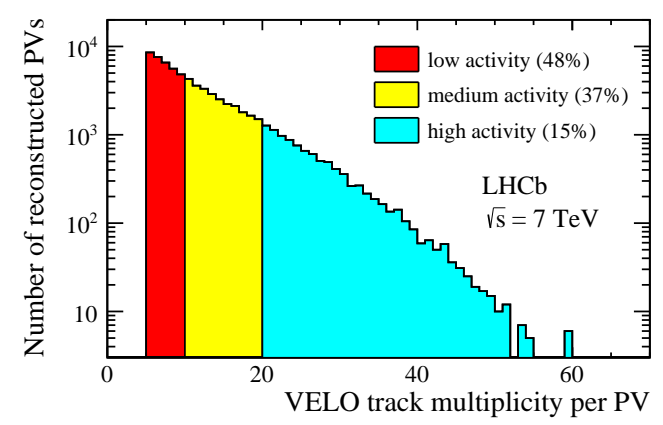

Figure 3: Multiplicity of reconstructed VELO tracks assigned to a PV. Different colours indicate three activity classes defined as fractions of the full distribution: red, from 5 to 10 tracks (low activity class); yellow, from 11 to 20 tracks (medium activity); blue, $\geq 21$ tracks (high activity) [11]. 

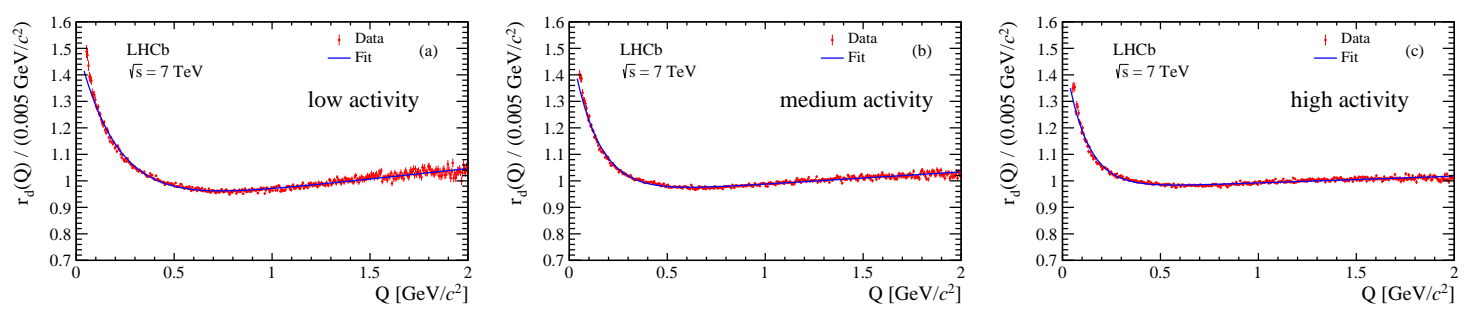

Figure 4: Results of the fit to the double ratio for like-sign pion pairs with event-mixed reference samples and the Coulomb effect subtracted for the three activity classes: (a) low, (b) medium and (c) high activity. Only statistical uncertainties are shown [11].

The fits to each multiplicity classes are shown in figure 4, while the best values for $R$ and $\lambda$ are shown in table 1. It is observed how the size of the emission source grows for higher multiplicity while chaoticity decreases. The same trend is observed at midrapidity at other LHC experiments $[15,16,17]$. The leading source of systematic uncertainty is due to differences in the event generators used to determine the double ratio and pile-up effects. Possible extensions to this study are double-differential studies and measurements in more complex systems such as $p \mathrm{~Pb}$ collisions.

\section{Prompt photon production in $\mathbf{p P b}$ at $\sqrt{s_{\mathrm{NN}}}=8.16 \mathrm{TeV}$}

Photons in $p \mathrm{~Pb}$ collisions can either be produced in hadronic decays or at the early stage of the collision. The later ones (called prompt or direct photons) are of great interest as they give direct sensitivity to the gluon parton distribution functions allowing to search for gluon saturation at low Bjorken- $x$ at forward rapidity. The LHCb acceptance allows to probe the region with $x<10^{-5}$ by measuring low- $p_{\mathrm{T}}$ photons in $p \mathrm{~Pb}$ collisions. In this section, the progress on this study is summarized, as presented in the $27^{\text {th }}$ International Conference on Ultrarelativistic Nucleus-Nucleus Collisions (Quark Matter 2018).

The LHCb strategy consists on finding the fraction of direct photons through measuring photons from hadronic decays. The results are given in term of a double ratio: $R_{\gamma}=\frac{\left(\gamma^{\text {inc }} / \gamma^{\pi^{0}}\right)_{\text {Data }}}{\left(\gamma^{\text {dec }} / \gamma^{\pi^{0}}\right)_{\mathrm{MC}}}$, where $\gamma^{\text {inc }}$ is the inclusive photon yield, $\gamma^{\text {dec }}$ is the yield of photons from hadron decays, and $\gamma^{\pi^{0}}$ is the yield of photons from $\pi^{0}$ decays. Excesses over unity would indicate the presence of direct photons. Note that the ALICE collaboration has measured direct photon production at low $p_{\mathrm{T}}$ in $\sqrt{s}=8$ and 2.76 TeV $p p$ collisions finding no significant direct photons signal [18].

The analysis is being conducted on the $\sqrt{s_{\mathrm{NN}}}=8.16 \mathrm{TeV} p \mathrm{~Pb}$ dataset acquired in 2016. Photons are reconstructed in the $e^{+} e^{-}$conversion channel, which offers better momentum resolution than photons directly reconstructed in the electromagnetic calorimeter (ECAL). The reconstructed photon fiducial region is limited to $2.5<\eta<4.0$ to avoid detector edge effects. The reconstructed converted photons yields are shown in figure 5 . These converted photons are combined with reconstructed photons in the ECAL clusters to reconstruct $\pi^{0}$ candidates, and a mass fit is used to extract the $\pi^{0}$ yield, as shown in figure 6. Differences in the efficiency of the ECAL between data and simulation must be taken into account, as well as the differences between the fraction of decay photons from $\pi^{0}$ decays in simulation and data. 

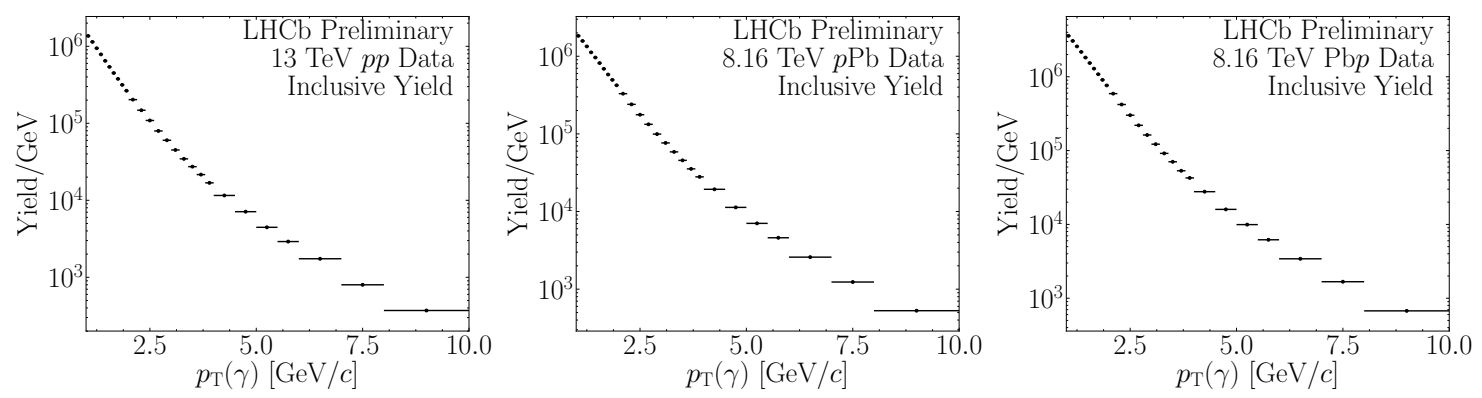

Figure 5: Inclusive converted photon yields for (left) $\sqrt{s}=13 \mathrm{TeV} p p$, (centre) $\sqrt{s_{\mathrm{NN}}}=8.16 \mathrm{TeV}$ $p \mathrm{~Pb}$, and (right) $\sqrt{s_{\mathrm{NN}}}=8.16 \mathrm{TeV} \mathrm{Pb} p$ data. By convention, in $p \mathrm{~Pb}$ data the proton beam heads toward the $\mathrm{LHCb}$ arm, while in $\mathrm{Pb} p$ data the lead beam heads towards the $\mathrm{LHCb}$ arm.
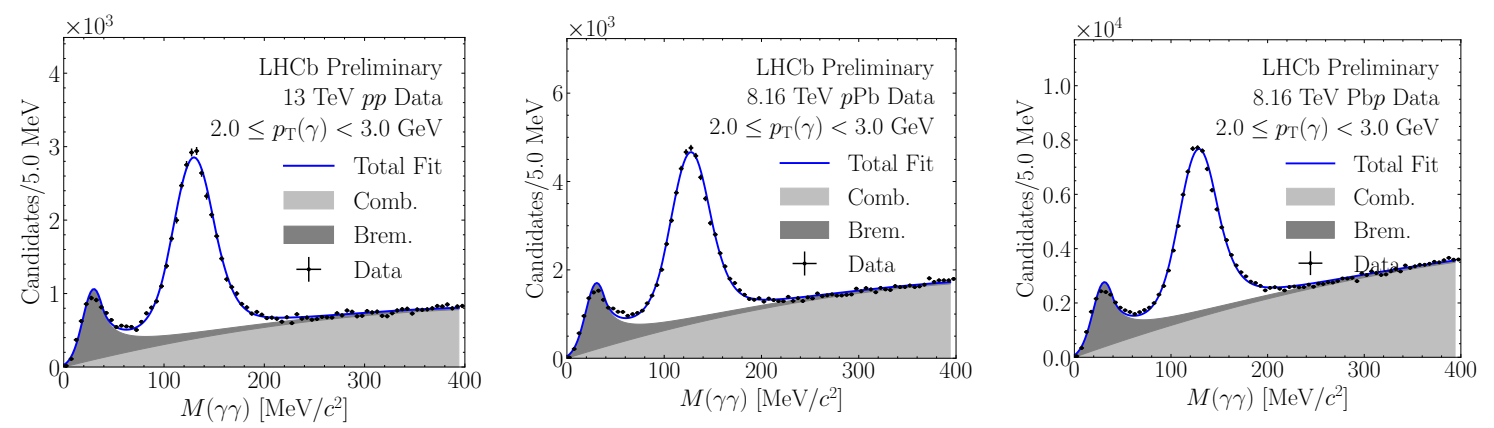

Figure 6: Sample mass distributions of diphoton candidates with one daughter reconstructed via its conversion products and the other reconstructed as an ECAL cluster for (left) $\sqrt{s}=13 \mathrm{TeV} p p$, (centre) $\sqrt{s_{\mathrm{NN}}}=8.16 \mathrm{TeV} p \mathrm{~Pb}$, (right) and $\sqrt{s_{\mathrm{NN}}}=8.16 \mathrm{TeV} \mathrm{Pb} p$ data in the $\pi^{0}$ mass region. Fit results are also shown. The total fit is shown as a solid line, while the combinatoric background is shown as a lightly shaded region. An additional background component resulting from the combination of a converted photon with a bremsstrahlung photon emitted by one of its daughters is shown as a darkly shaded region.

\section{Conclusions and prospects}

A diversity of measurements related to soft-particle production in heavy-ion physics at $\mathrm{LHCb}$ have been reported, exemplifying the expansion of the heavy-ion physics program at LHCb. With regard to antiproton production in $p \mathrm{He}$ collisions, it constitutes a unique measurement with valuable inputs for the cosmic ray community, and it is the first measurement with LHCb operating in its fixed-target configuration. The measurement of the Bose-Einstein correlations in $p p$ allowed to confirm the results from other experiments at forward rapidity, and opened a door to further studies in $p \mathrm{~Pb}$ collisions. Finally, prompt photon production at $\mathrm{LHCb}$ is showing promising progress and giving expectations on shedding more light on the gluon saturation panorama. These results show how the LHCb potential in heavy-ion physics is yet to be fully exploited. 


\section{References}

[1] LHCB collaboration, R. Aaij et al., LHCb Detector Performance, Int. J. Mod. Phys. A30 (2015) 1530022 [1412.6352].

[2] LHCB collaboration, R. Aaij et al., Precision luminosity measurements at LHCb, JINST 9 (2014) P12005 [1410.0149].

[3] AMS collaboration, M. Aguilar et al., Antiproton Flux, Antiproton-to-Proton Flux Ratio, and Properties of Elementary Particle Fluxes in Primary Cosmic Rays Measured with the Alpha Magnetic Spectrometer on the International Space Station, Phys. Rev. Lett. 117 (2016) 091103.

[4] O. Adriani et al., Measurement of the flux of primary cosmic ray antiprotons with energies of 60-MeV to 350-GeV in the PAMELA experiment, JETP Lett. 96 (2013) 621.

[5] LHCB collaboration, R. Aaij et al., Measurement of antiproton production in $\mathrm{pHe}$ collisions at $\sqrt{s_{N N}}=110 \mathrm{GeV}, 1808.06127$.

[6] T. Pierog, I. Karpenko, J. M. Katzy, E. Yatsenko and K. Werner, EPOS LHC: Test of collective hadronization with data measured at the CERN Large Hadron Collider, Phys. Rev. C92 (2015) 034906 [1306.0121].

[7] T. Pierog and K. Werner, EPOS Model and Ultra High Energy Cosmic Rays, Nucl. Phys. Proc. Suppl. 196 (2009) 102 [0905.1198].

[8] M. Gyulassy and X.-N. Wang, HIJING 1.0: A Monte Carlo program for parton and particle production in high-energy hadronic and nuclear collisions, Comput. Phys. Commun. 83 (1994) 307 [nucl-th/9502021].

[9] T. Sjöstrand, S. Mrenna and P. Z. Skands, PYTHIA 6.4 Physics and Manual, JHEP 05 (2006) 026 [hep-ph/0603175].

[10] S. Ostapchenko, Monte Carlo treatment of hadronic interactions in enhanced Pomeron scheme: I. QGSJET-II model, Phys. Rev. D83 (2011) 014018 [1010 . 1869].

[11] LHCB collaboration, R. Aaij et al., Bose-Einstein correlations of same-sign charged pions in the forward region in pp collisions at $\sqrt{s}=7$ TeV, JHEP 12 (2017) 025 [1709.01769].

[12] T. Kanki, K. Kinoshita, H. Sumiyoshi and F. Takagi, Multiparticle Production in Particle and Nuclear Collisions. 1, Prog. Theor. Phys. Suppl. 97A (1988) 1.

[13] S. Pratt, Coherence and Coulomb Effects on Pion Interferometry, Phys. Rev. D33 (1986) 72.

[14] T. Csorgo, S. Hegyi and W. A. Zajc, Bose-Einstein correlations for Levy stable source distributions, Eur. Phys. J. C36 (2004) 67 [nucl-th/0310042].

[15] CMS collaboration, V. Khachatryan et al., Measurement of Bose-Einstein Correlations in pp Collisions at $\sqrt{s}=0.9$ and 7 TeV, JHEP 05 (2011) 029 [1101.3518].

[16] ALICE collaboration, K. Aamodt et al., Femtoscopy of pp collisions at $\sqrt{s}=0.9$ and 7 TeV at the LHC with two-pion Bose-Einstein correlations, Phys. Rev. D84 (2011) 112004 [1101. 3665].

[17] ATLAS collaboration, G. Aad et al., Two-particle Bose-Einstein correlations in pp collisions at $\sqrt{\mathbf{s}}=$ 0.9 and 7 TeV measured with the ATLAS detector, Eur. Phys. J. C75 (2015) 466 [1502 . 07947 ].

[18] ALICE collaboration, S. Acharya et al., Direct photon production at low transverse momentum in proton-proton collisions at $\sqrt{\mathbf{s}}=\mathbf{2 . 7 6}$ and $8 \mathrm{TeV}, 1803.09857$. 